\title{
A Case Study of Double Lid Driven Cavity for Low Reynolds Number Flow
}

\author{
Doli Rani Pal ${ }^{1 *}$, Goutam Saha ${ }^{1,3}$ and Kajal Chandra Saha ${ }^{2}$ \\ ${ }^{I}$ Department of Mathematics, Dhaka University, Dhaka-1000, Bangladesh \\ ${ }^{2}$ Department of Applied Mathematics, Dhaka University, Dhaka-1000, Bangladesh \\ ${ }^{3}$ Department of Mathematics and Statistics, York University, Toronto, ON M3J 1P3, Canada
}

(Received: 9 November 2017 ; Accepted: 24 May 2018)

\begin{abstract}
The present numerical study has been accomplished remembering the true objective to explore the low Reynolds number flow with heat exchange behaviors inside a double lid driven square shaped cavity loaded with fluids such as air and water. Specifically, this work explores laminar, steady, incompressible mixed convection flow inside the cavity which has been done numerically by applying Finite Difference Method (FDM). In this analysis, top wall is treated as the heated wall and remaining walls are considered as cold walls. In addition, vertical side walls are moving at a relentless speed in its own particular plane along with the positive direction. Moreover, the numerical simulation is being conveyed out to observe the behavior of flow and thermal fields for variation of low Reynolds Number (Re), Richardson number (Ri) and Prandtl number(Pr). Also, the results have been validated through a comparison with other research works and a good agreement is observed. Also, results show that the above mentioned dimensionless parameters have significant effects on the flow and heat exchange attributes. Moreover, the effects of these parameters on average Nusselt number $(\mathrm{Nu})$ have been analyzed and presented.
\end{abstract}

Keywords: Heat transfer, Reynolds number, Nusselt number, Richardson number, Prandtl number.

\section{Introduction}

The double lid driven square cavity plays a compelling role in controlling the flow phenomena. It is additionally helpful in mechanical and designing field. Over the last few decades, the double lid-driven cavity problem has been gained extensive interest among the scientists and researchers because of its simplest geometrical settings with all fluid mechanical structure and applications such as cooling of electronic gadgets, drying instrument, covering, softening procedures and so forth. Different case study has been done by considering the physical model and also these types of application in which the horizontal top ${ }^{1-4}$ or bottom wall ${ }^{5}$ sliding lid-driven two dimensional cavity or inclined $^{6}$ at an angle and all has been moving with a constant velocity. Also the study has been carried out in which the side walls ${ }^{7}$ were moving. In these studies, various boundary conditions has been applied and to make a temperature gradient in the cavity normally the lid-driven side and the one restricting are warmed differentially. The application of multi-grid method in the solution of NavierStokes equation has been analysed by Ghia et al. ${ }^{8}$ with a goal of obtaining solutions for higher Reynolds number and mesh refinements. It has been studied the solutions for incompressible flow using the Navier-Stokes equations considering high $R e$ in which the horizontal top wall has been moving. Also observed that fine-mesh solution are obtained for high $R e(100 \leq R e \leq 10,000)$ flow by using the coupled strongly implicit and multi-grid methods. By the motivation of the application in the production of molten metal, Moallemi et al. ${ }^{9}$ has been revealed Prandtl number effects $(0.01<P r<50)$ on laminar mixed transmission heat transfer in a lid driven cavity. They concluded that because of higher Prandtl number $(P r)$, there exists buoyancy force on the heat and mass exchange inside the cavity and mixed convection arises by the mix of buoyancy forces due to temperature distinction and forced convection. Hakan et al. ${ }^{10}$ carried out numerical investigation of double lid-driven enclosure for different Richardson number and considered cases of mixed, forced region. Chamkha et al. ${ }^{11}$ have demonstrated hydrodynamic flow behaviour inside a enclosure with heat production or ingestion where left side wall was moving. He examined that for a fixed value $G r$, average Nusselt number increments with the expansion of $R e$ for both aiding and contradicting conditions. Alternatively, average Nusselt number increments only for aiding flow. In recent years, there has been remarkable enthusiasm in studying the inclination of square cavity loaded with nanofluid because the impacts of the inclined cavity assume a vital part of cooling of electronic devices particularly inside the PCs. these sorts of PCs work at various inclination angles and in various conditions. Researchers ${ }^{12-14}$ have discovered that the inclination of the cavity is more energetic in natural convection than the forced convection. The parameters $\mathrm{Gr}$ and $R e$ can be grouped into single parameter $R i$ and this has been gained interest among researchers. Also it has been observed that the average Nusselt number is surged with the inclined cavity. Cheng et al. ${ }^{15}$ identified the heat transfer characteristics inside a square enclosure with different Richardson and Prandtl numbers. It is discovered that there is no bifurcation happened for the scopes of $\operatorname{Re}(10 \leq R e \leq 2200)$ and $\operatorname{Pr}(\operatorname{Pr}=0.01,0.71,6.0,50)$ but when $R i=1$ and 10, the flows experience a Hopf bifurcation for certain Reynolds Number. A point by point study of lid-driven cavity flow at moderate Reynolds number has been studied by Khorasanizade et al. ${ }^{16}$ and numerical investigation of a mixed convection flow in a lid-driven cavity problem build upon the aspect ratio studied by Omari et al. ${ }^{17}$. This investigation showed that for smaller cavity aspect ratio an energy rate enhances due

*Author for correspondesnce. e-mail: doli.math@du.ac.bd 
to circulation movements. A basic understanding strategy has been created by Adair et al. ${ }^{18}$ for solving Navier Stokes equation by utilizing limited contrast technique in a lid driven cavity where the top wall is moving. They have utilized vorticity streamline equation to solve NavierStokes equation and got an exceptionally more straightforward strategy to depict the flow phenomena. Considering the conceivable four instances of moving velocity contingent upon the wall, Malik et al. ${ }^{19}$ have demonstrated a comparative investigation of mixed convection fluid inside a square cavity. It has been built up that the heat transfer and fluid flow is delicate to the solid volume portion. Every one of these examinations have been considered for high Reynolds number. So, in this study, research is carried out for low Reynolds number flow.

Number of concentrates throughout the years was led under thought of mixed convection heat exchange attributes of a square molded cavity. In such manner, the goal of this investigation is to review the behavior of fluid and heated fields for variety of low Reynolds Number $(R e)$ in a double lid driven square cavity. Likewise investigate the impacts of Prandtl and Richardson number in the cavity where the side walls (left and right) are moving at a consistent speed and top wall is considered as heated wall. Moreover, average Nusselt number behaviour is investigated in order to see the performance of heat transfer inside the cavity.

\section{Model Illustration and Governing Equation}

The physical model and boundary conditions are appeared in Fig.1 which is double lid driven square cavity loaded with fluids such as air and water. Fluid flow and heat transfer phenomena are described inside the cavity where the vertical walls are moving at a steady speed $V_{L}$ and the top wall is kept up isothermally at temperature $T_{H}$. In this study, remaining walls are considered as cold walls.

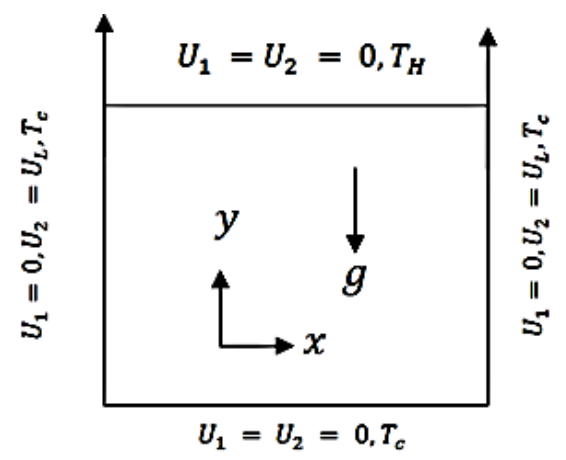

Fig. 1. Schematic description of the physical model.

The flow phenomena is assumed to be steady, laminar and the fluid is treated as Newtonian, incompressible and mixed convective likewise ignoring the viscous dissipation impact. The accompanying governing equation has been displayed for two-dimensional laminar and incompressible stream in non-dimensional shape:

$$
\begin{aligned}
& \frac{\partial U_{1}}{\partial X_{1}}+\frac{\partial U_{2}}{\partial X_{2}}=0 \\
& U_{1} \frac{\partial U_{1}}{\partial X_{1}}+U_{2} \frac{\partial U_{1}}{\partial X_{2}}=-\frac{\partial P}{\partial X_{1}}+\frac{1}{R e}\left(\frac{\partial^{2} U_{1}}{\partial X_{1}{ }^{2}}+\frac{\partial^{2} U_{1}}{\partial X_{2}{ }^{2}}\right) \\
& U_{1} \frac{\partial U_{2}}{\partial X_{1}}+U_{2} \frac{\partial U_{2}}{\partial X_{2}}=-\frac{\partial P}{\partial X_{2}}+\frac{1}{R e}\left(\frac{\partial^{2} U_{2}}{\partial X_{1}{ }^{2}}+\frac{\partial^{2} U_{2}}{\partial X_{2}{ }^{2}}\right) \\
& +R i \theta \\
& U_{1} \frac{\partial \theta}{\partial X_{1}}+U_{2} \frac{\partial \theta}{\partial X_{2}}=\frac{1}{\operatorname{Pr} R e}\left(\frac{\partial^{2} \theta}{\partial X_{1}^{2}}+\frac{\partial^{2} \theta}{\partial X_{2}^{2}}\right)
\end{aligned}
$$

where $U_{1}$ and $U_{2}$ are the non-dimensional velocity parts along $X_{1}$ and $X_{2}$ bearings, $\theta$ is the non-dimensional temperature and $P$ is the non-dimensional pressure term. Here, the dimensionless parameters in Eqs. (1-4) are: $R i$ is the Richardson number, $R e$ is the Reynolds number, $G r$ is the Grashof number, $P r$ is the Prandtl number. The governing parameters in Eqs. (1-4) and non-dimensional length and velocities are presented as follows:

$$
\begin{gathered}
X_{1}=\frac{x}{L} ; X_{2}=\frac{y}{L} ; U_{1}=\frac{u}{U_{L}} ; U_{2}=\frac{v}{U_{L}} ; P=\frac{p}{\rho U_{L}{ }^{2}} ; \theta \\
=\frac{T-T_{C}}{T_{H-} T_{C}} ; \\
R i=\frac{G r}{R e^{2}} ; R e=\frac{V_{L} L}{v} ; G r=\frac{g \beta \Delta T L^{3}}{v^{3}} ; \operatorname{Pr}=\frac{v}{\alpha} ;
\end{gathered}
$$

where $\rho, g, \alpha, \beta$ and $v$ are the fluid density, gravitational acceleration, thermal diffusivity coefficient of volumetric expansion and kinematic viscosity respectively. The velocity and temperature flow field with boundary conditions are shown in Fig.1 and have the resulting dimensionless form:

$$
\begin{array}{cc}
U_{1}=0, U_{2}=1, \theta=0 & \text { at } X_{1}=0 \text { and } X_{1}=1 \\
U_{1}=U_{2}=0, \theta=0 & \text { at } X_{2}=0 \\
U_{1}=U_{2}=0, \theta=1 & \text { at } X_{2}=1
\end{array}
$$

Also, the local and average Nusselt number are defined as

$$
\begin{gathered}
N u(c)=-\left.\frac{\partial \theta}{\partial X_{2}}\right|_{X_{2}=1} \\
\overline{N u}=\int_{0}^{1} N u(c) d c
\end{gathered}
$$

It is noted that Eq. (6) is calculated using forth order onesided approximation and Eq. (7) is integrated using Simpson's $\frac{1}{3}$ rule.

\section{Numerical Methodology and Comparison}

The dimensionless governing equations together with the boundary circumstances (1-5) have been discretised and then figured out numerically by using Finite difference method (FDM) and in house code named DGK is used for meshing, simulation and post-processing using Mathematica 9.0 with Intel ${ }^{\circledR}$ Core $^{\mathrm{TM}}$ i3-4160T CPU @ 3.10 $\mathrm{GHz}$ processor, 4 GB RAM and 64-bit operating system. Numerical simulations have been carried out for different uniform grid sizes such as $41 \times 41,61 \times 61,81 \times 81$ and 
presented in Table 1. And a grid independent result is observed for $61 \times 61$ grid size, therefore $61 \times 61$ uniform grids are considered throughout the simulation. Moreover, a double lid-driven cavity loaded with air/water is considered in which the upper wall is inspected as hot wall and lower wall as cold wall. Also the side walls are moving at a constant speed in the same positive direction. The outcomes for flat $U_{1}$-velocity and vertical $U_{2}$-velocity are looked at for $R i=0.0, R e=100$ and 400, $P r=0.71$, and outcomes are discovered great concurrence with the outcomes of Adair et al. ${ }^{18}$ which is appeared in Fig. 2 and Ghia et al. ${ }^{8}$ which is appeared in Fig. 3.
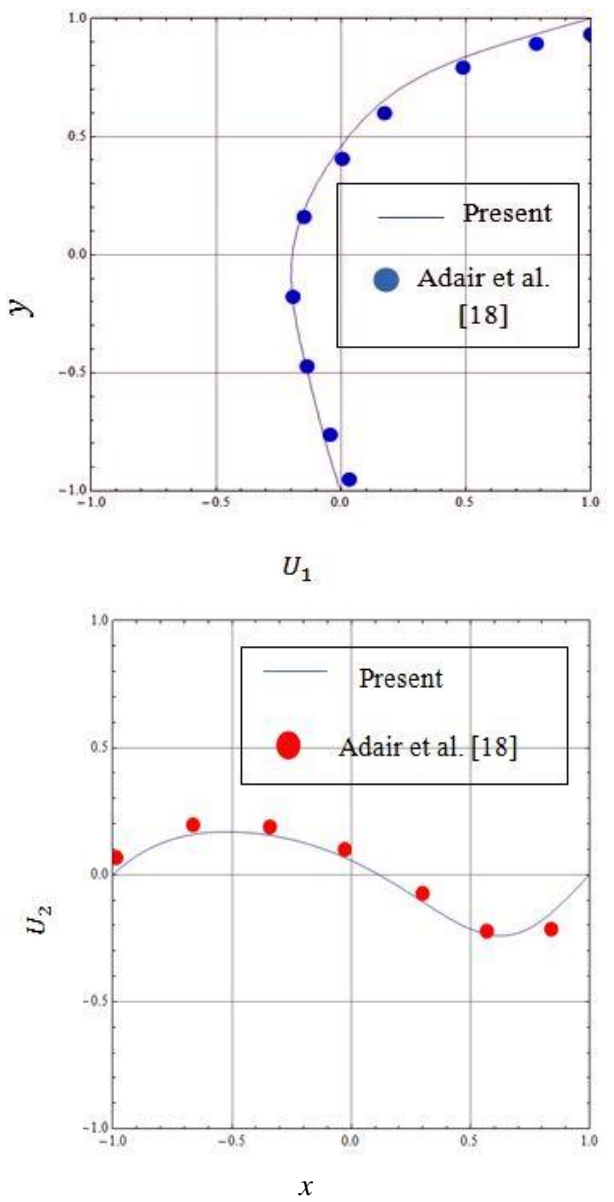

Fig. 2. Velocity profiles along vertical (up) and horizontal (down) centerlines for $R e=100$ in comparison with the result of Adair et $a l .{ }^{18}$ (Here, $x$ indicates $x_{1}$ and $y$ indicates $x_{2}$ ).

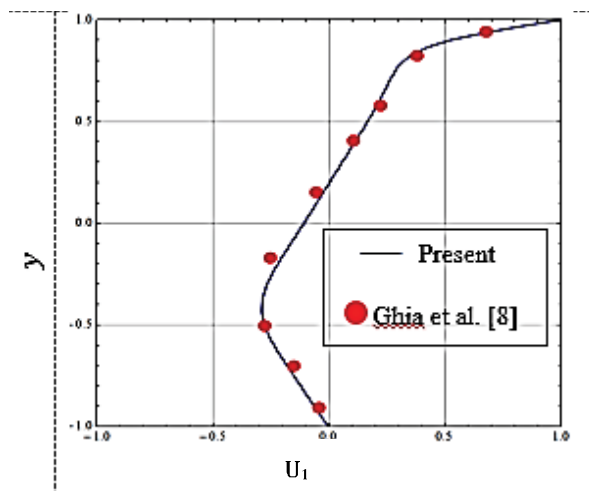

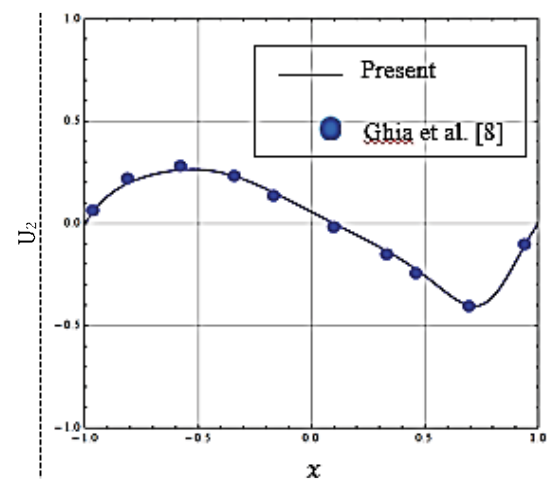

Fig. 3.Velocity profiles along vertical $\left(1^{\text {st }}\right)$ and horizontal $\left(2^{\text {nd }}\right)$ centerlines for $R e=400$ in comparison with the result of Ghia et $a l .{ }^{8}$ (here, $x$ indicates $x_{1}$ and $y$ indicates $x_{2}$ ).

Another examination has been led for average Nusselt number to approve the code. An average Nusselt Number correlation is made with Abu-Nada et al. ${ }^{6}$, Waheed et $a l .^{4}$ and Sharif et $a l .{ }^{14}$, as appeared in Table 2 for the reaches $1 \leq R e \leq 1000$ and $G r=100$. Comparing our results with those results, it can be concluded that the agreement is found to be good to continue the present research.

Table 1. Grid independence test for $\operatorname{Pr}=0.71, R i=$ 1. $0, R e=500$ by using present code

$\begin{array}{cc}\text { Grid size } & \text { Average } N u \\ 41 \times 41 & 9.06 \\ 61 \times 61 & 9.46 \\ 81 \times 81 & 10.09\end{array}$

Table 2. Validate average Nusselt number at the lid surface for $\boldsymbol{G r}=100$ by using present code

\begin{tabular}{cccccc}
\hline$R e$ & $R i$ & $\begin{array}{c}\text { Avg Nu } \\
\text { current } \\
\text { work } \\
61 \times 61\end{array}$ & $\begin{array}{c}\text { Abu-Nada } \\
\text { et al. [6] } \\
61 \times 61\end{array}$ & $\begin{array}{c}\text { Waheed } \\
{[4]}\end{array}$ & $\begin{array}{c}\text { Sharif } \\
{[14]}\end{array}$ \\
\hline 1 & 100 & 1.00 & 1.006367 & 1.00033 & --- \\
\hline 100 & 0.01 & 2.05 & 2.112957 & 2.03116 & --- \\
\hline 400 & 0.000625 & 3.83 & 4.069035 & 4.02462 & 4.05 \\
\hline 500 & 0.0004 & 4.25 & 4.602600 & 4.52671 & --- \\
\hline 1000 & 0.0001 & 6.15 & 6.426109 & 6.48423 & 6.55 \\
\hline
\end{tabular}

\section{Results and Discussion}

In this study, convective flow in a double lid driven cavity has been contemplated for the impact of heat transfer in which the vertical walls are moving at a consistent speed. Also, fluid inside the cavity is considered to be air/water. The dimensionless parameters are Reynolds number, Richardson number and Prandtl number.

Considering ranges are $100 \leq R e \leq 500,0.3 \leq R i \leq 10$ and $0.71 \leq \operatorname{Pr} \leq 8.0$.

\section{Effect of Reynolds Number and Richardson Number}

In Fig. 4. (a, e, i), the left and right geometry show the velocity and temperature profiles for four different cases of $R e$ for $R i=0.3$. Form all the velocity profiles, it can be 
seen that they are separated in two different direction along the centerline $x=0.5$. From Fig. 4. (a), it is observed that for $R i=0.3$ as $R e$ is increasing the fluid circulated vortices are moving upward in counter clock-wise direction supporting the shear effect as compared to buoyancy effects and magnitude of velocities are decreasing. And it remains same for the different Reynolds number which is demonstrated in Fig. 4 (e, i). The temperature profiles are presented in Fig. 4(a, e, i) for $R e=100$ and $R i=0.3$ and seen that in the first figure the shape is parabolic and it becomes twisted near the vertical wall when the Reynolds number increases. And also said that for low Reynolds number the flow is controlled by buoyancy force. From Fig. $4(\mathrm{~b}, \mathrm{f}, \mathrm{j})$ for various values of $R e$ with $R i=0.3,1.0,5.0$, the velocity profiles is also divided along the centerline $x=$ 0.5 . From Fig 4(b), it is seen that the velocity profile is same as velocity profile of Fig. 4(a) for $R i=1.0$ and $R e=100$ and the temperature profile represents parabolic behavior. From Fig. 4 (f, j), when the Reynolds numbers enhance with the increase of Richardson number, the velocity profile is divided into four parts. The primary vortex has been shifted near the side wall and there appeared another secondary vortex near the centerline. After that the temperature profiles for that geometry becomes stable and parallel to the lower wall. Also form Fig. 4 (c, d, g, h, k, 1), when $R e$ increases with $R i=$ $5.0,10.0$, the opposite behavior is visible for buoyancy effect. It is discovered that with the increase of Reynolds number $(R e)$, the cold fluid parallel to as far as possible constrained to move profoundly region of gap and warmed liquid advances toward as far as possible to fill the bit. Thus warm exchange is limited at higher Reynolds number. The flows totally constrained overwhelmed at bigger Reynolds number.

It has been shown that the velocity profile for low Richardson numbers creates two secondary vortexes and also exists for $R i=1.0$ and all the profiles divided along the centerline $x=0.5$ but when Richardson number $(R i)$ increases, the number of vortex increases which has been shown in right geometry of each case for different Richardson number. For $R e=100$ in Fig. 4(a,b,c,d), it has been observed that on increasing of $R i$ values from 0.3 to 10.0 , the buoyancy effect is visible clearly from the evaluation of velocity profiles. Also from Fig. 4(e, f, g, h) and Fig. 4(i, j, k, l), it has been shown that the velocity profiles with the increase for Richardson number $(R i)$, the stream function value increases in core of the cavity near the sliding wall for higher Reynolds number $(R e)$. Thus the shear effect is dominated by buoyancy effect. The temperature profiles Fig. 4(a, b, c, d) (left geometry) shows that the heat plums arise below each heat source. Buoyancy forces force the heated fluid to enter into the cold region from heat source and lower cold fluid move upward. Then with the increase of $R i$, the heat transfer rate decreases. From Fig. 4 (all right geometry) similar configurations for temperature profiles are observed for higher buoyancy forces. For higher $R i$, the temperature profiles are gathered close to sliding wall. Finally, average $\mathrm{Nu}$ results for different values of $R e$ and $R i$ when $P r=0.71$ have been presented in Table 3.

\section{Effect of Prandtl Number}

Fig. 5 represents the velocity (left) and temperature (right) profiles for various Prandtl's number ((a). $P r=0.71$, (b). $P r=2.0$, (c). $\operatorname{Pr}=4.0$ and (d). $P r=8.0$ )with $R e=500$, $R i=5.0$. From Fig. 5(a), it is seen that the velocity profiles are divided into two parts along the centerlines. It is observed that inside the cavity there appears primary and secondary vortex and primary vortexes flatten near two side walls. Then from the velocity profiles of Fig. 5(b, c, d), it is also observed that the secondary vortex creates so many sub vortex and it increases with the increment of Prandtl number. Altogether, it looks like a shape of a garlic along the centerline. So, with the increase of Prandtl number due to buoyant effect, the fluid circulates in counter clockwise from the left side of the cavity and clockwise in the right side of cavity near the sliding walls. Also the temperature difference between upper hot wall and cool bottom and side walls, the secondary vortex increases in number in the core region of the cavity and become shrinked. From Fig. 5(a, b, c, d), the temperature profiles represent that with the increase of Prandtl number the cold fluid moves to the hot surface. The velocity dissemination gives a thin thermally influenced area, making the warm impact be kept to this thin region adjoining the warmed wall. For this reason, the velocity profile for higher Prandtl number liquids approaches the forced convection mode. From Fig. 5 for fluids with a lower Prandtl number, the thermally influenced region grows from the hot wall to cool wall thus does the region that is influenced. Finally, the average Nusselt number increases with the increase of Prandtl number which has been presented in Table 4 .

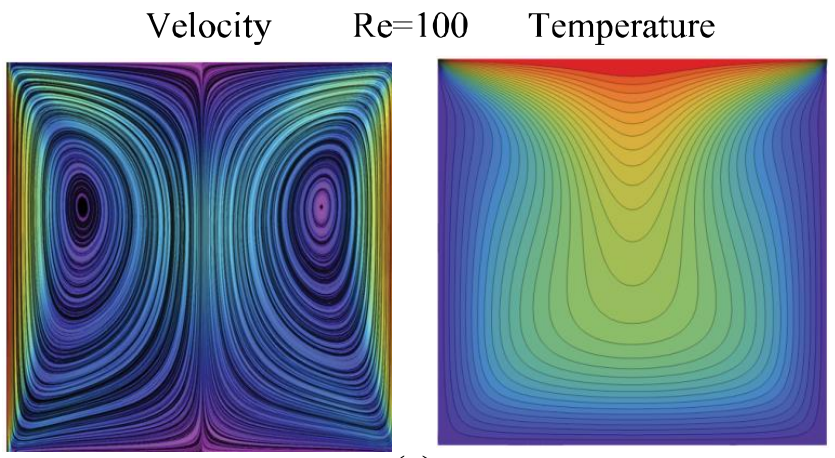

(a)
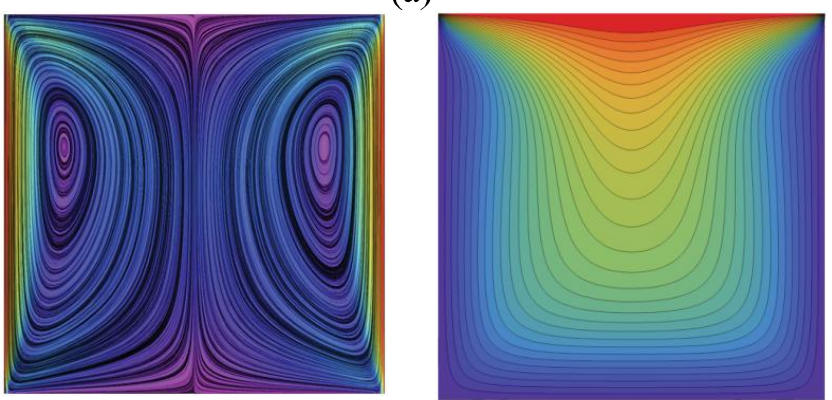

(b) 

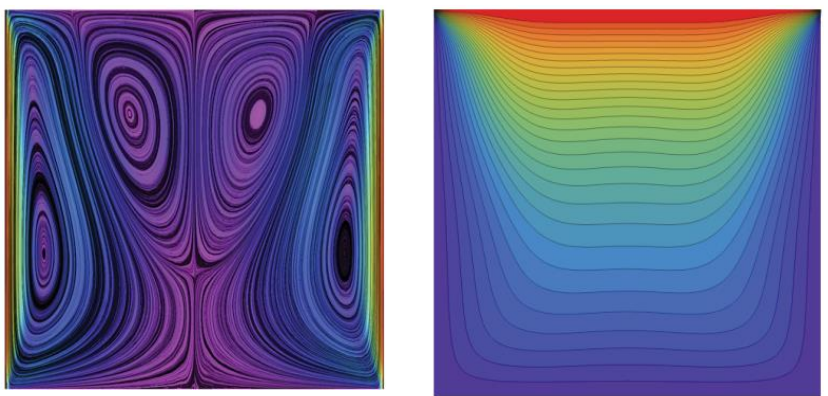

(c)
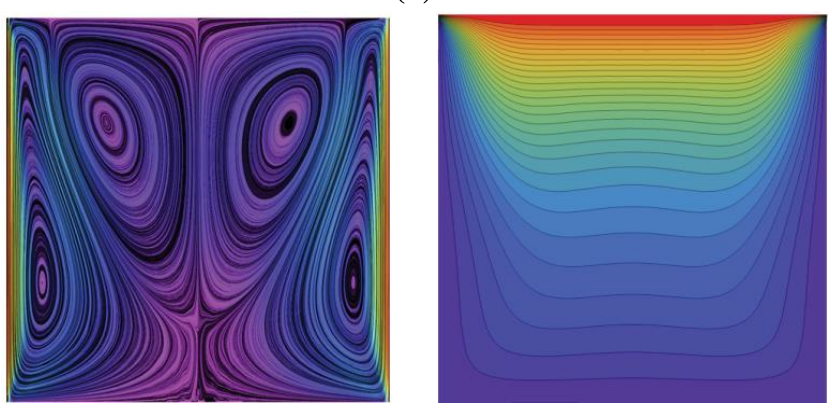

(d)

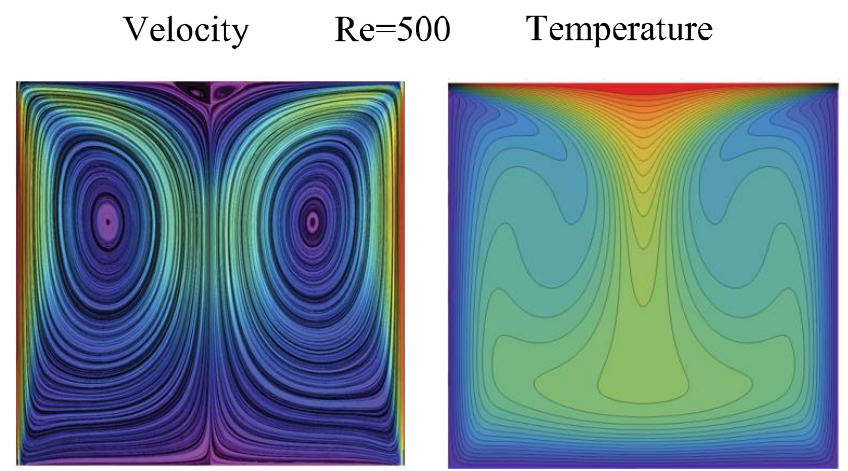

(e)
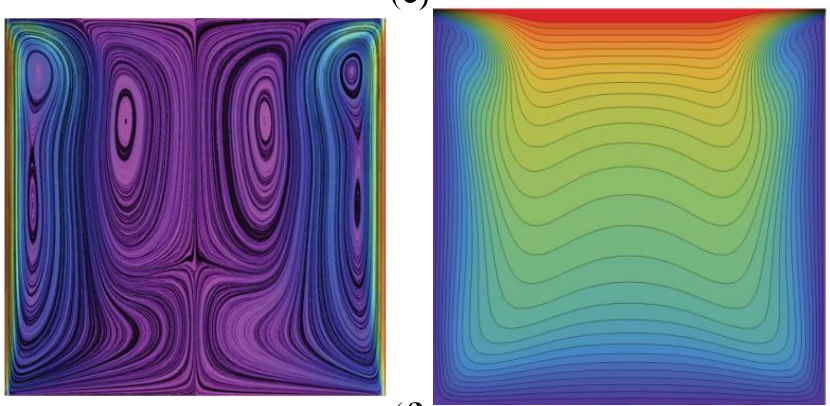

(f)
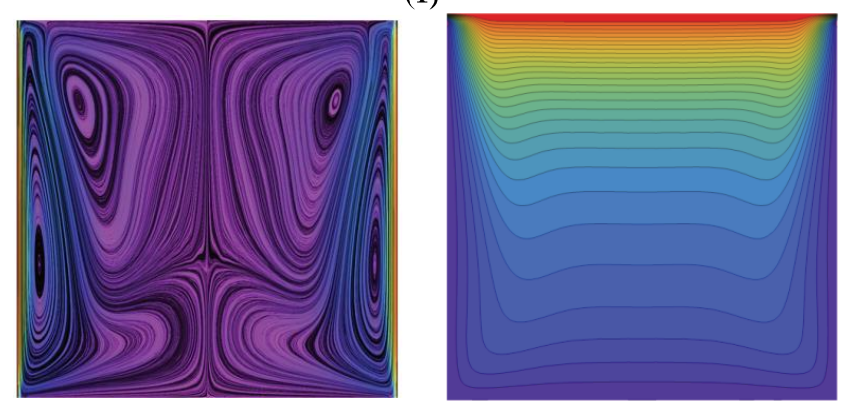

(g)
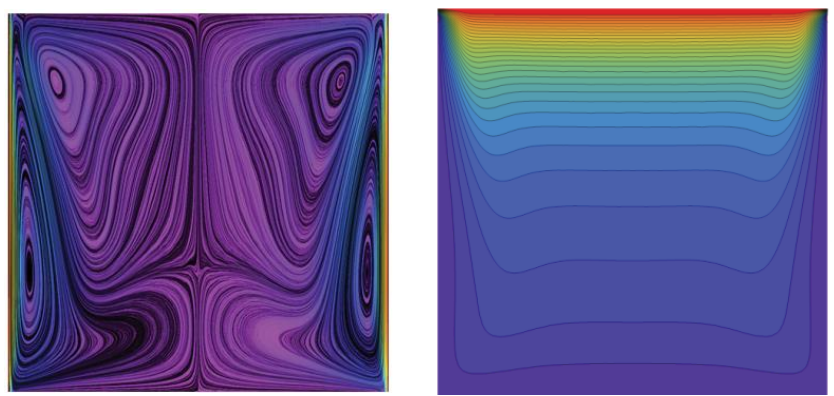

(h)

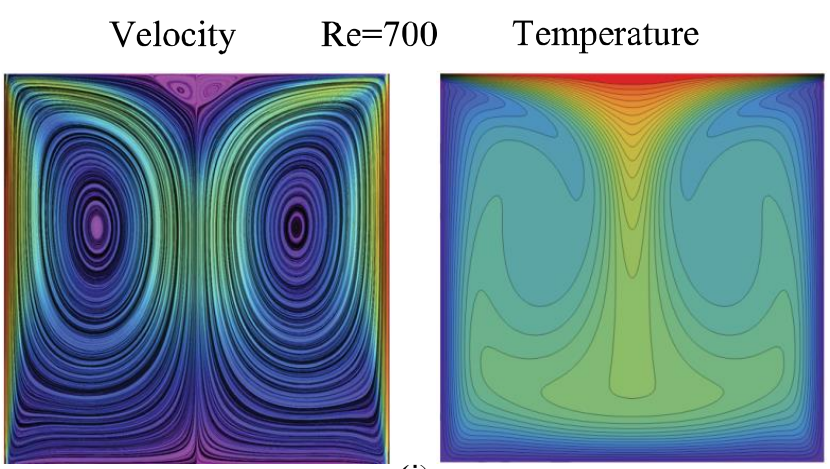

(i)
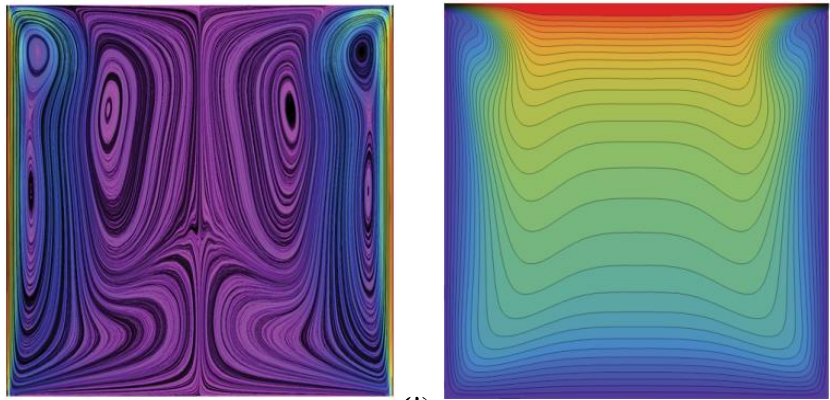

(j)
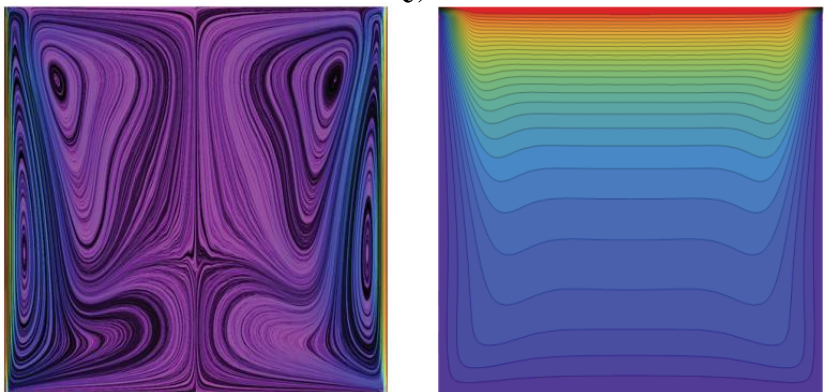

(k)
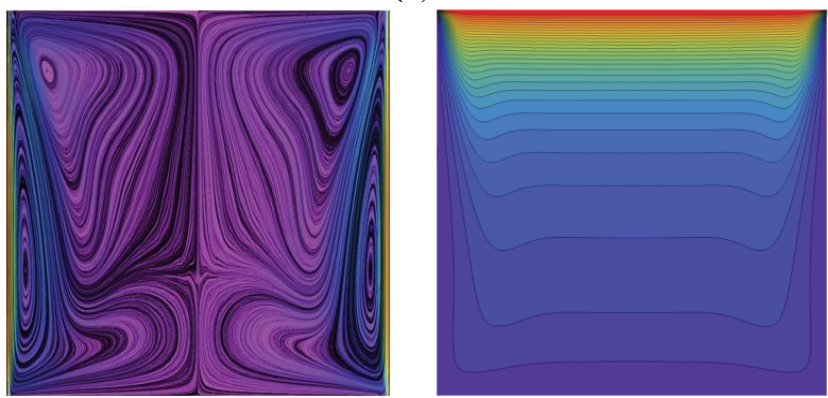

(l)

Fig. 4. Deviation of Velocity and Temperature profiles for various Richardson's number, $R i$ with $(a, e, i) R i=0.3,(b, f, j) R i=1.0$, $(c, g, k) R i=5.0$ and $(d, h, l) R i=10.0$ with $\operatorname{Pr}=0.71$. 

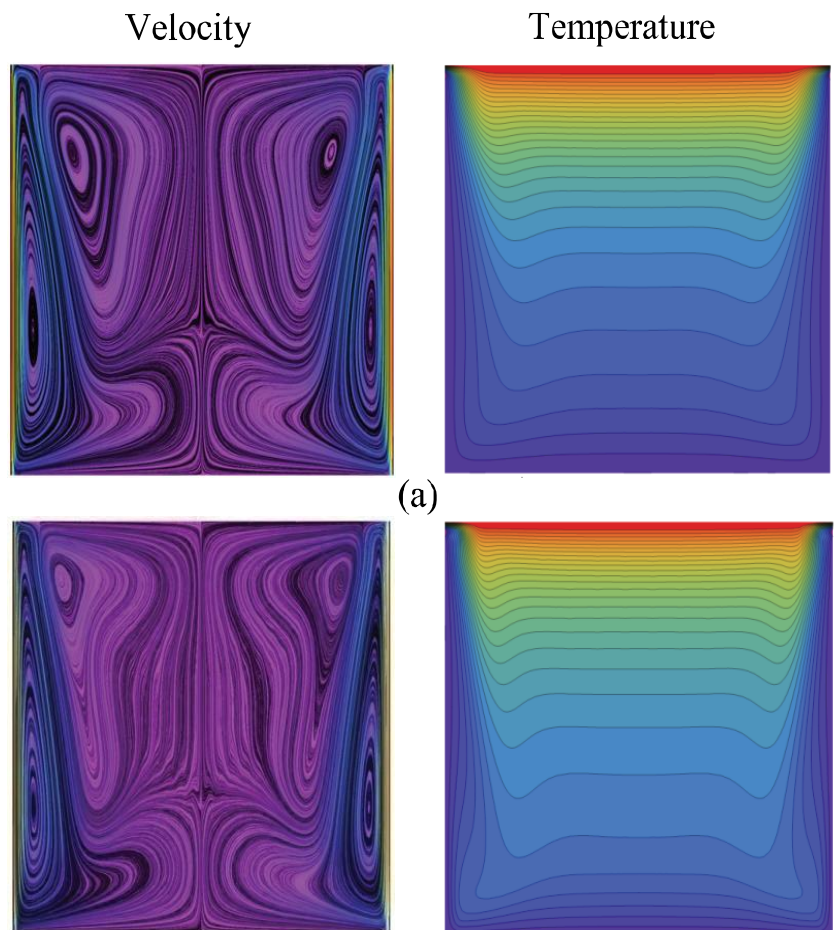

a)

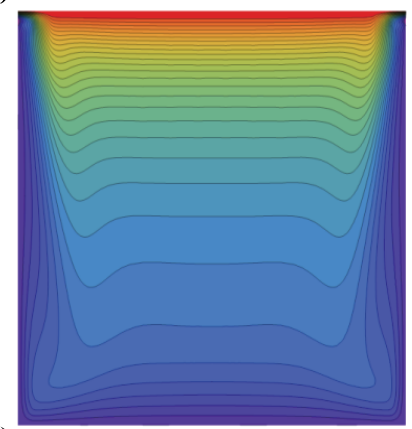

(b)
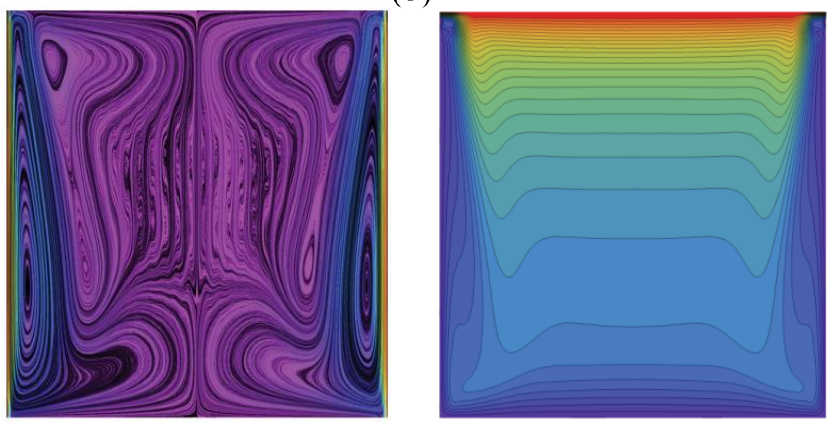

(c)
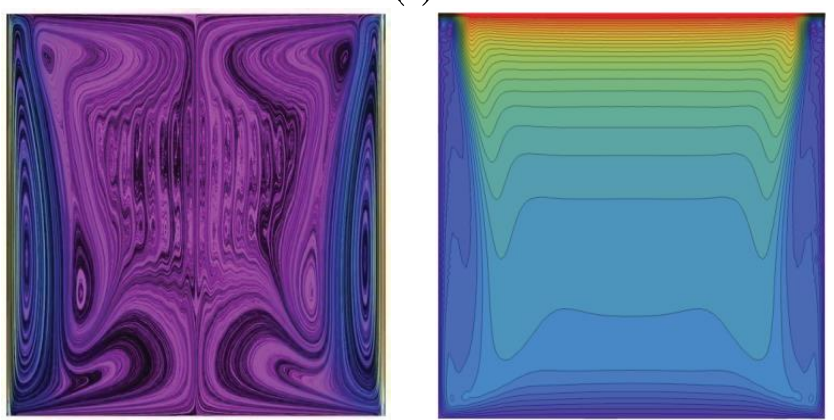

(d)

Fig. 5. Deviation of velocity and temperature profiles for different Prandtl's number, $\operatorname{Pr}$ (a) $\operatorname{Pr}=0.71$, (b) $\operatorname{Pr}=2.0$, (c) $\operatorname{Pr}=4.0$ and (d) $P r=8.0$ when $\mathrm{Re}=500, \mathrm{Ri}=5.0$.

Table 3. Average Nusselt number when $\operatorname{Pr}=\mathbf{0 . 7 1}$

Average Nusselt Number, $\mathrm{Nu}$

$\begin{array}{ccccc}\mathrm{Re} & \mathrm{Ri}=0.3 & 1.0 & 5.0 & 10.0 \\ 100 & 7.33 & 6.79 & 5.74 & 5.70 \\ 500 & 13.12 & 9.46 & 6.46 & 6.53 \\ 700 & 15.06 & 10.37 & 6.68 & 6.86\end{array}$

Table 4. Average $\mathrm{Nu}$ results for different values of $\operatorname{Pr}$ with $R e=500 \& R i=5$.

\begin{tabular}{cc}
\hline$P r$ & Avg. $\mathrm{Nu}$ \\
\hline 0.71 & 6.46 \\
\hline 2.0 & 7.74 \\
\hline 4.0 & 8.81 \\
\hline 8.0 & 9.88 \\
\hline
\end{tabular}

\section{Conclusions}

This work explores laminar, steady, incompressible, mixed convection flow inside the cavity filled with air or water in a lid driven square shaped compound was defined and explained numerically utilizing a 4th-order Finite Difference Method (FDM). The technique was approved the past distributed work considering their cases and results are observed to be great in arrangement. Graphical outcome for different dimensionless parameters were exhibited and discussed. From the above graphical presentation this can conclude the following assumptions:

- The heat transfer rate decreases with the increment of Richardson number $(R i)$.

- The effects of Reynolds number on the flow field is dominated by forced convection. When the Reynolds number $(R e)$ rises, the heat transfer rate decreases.

- The impact of buoyancy on the flow and heat transfer in the cavity is observed to be more noticeable for higher estimations of $\mathrm{Pr}$, if $R e$ and $\mathrm{Ri}$ are kept consistent. Likewise the heat transfer rate is expanding with the expansion of Prandtl number.

\section{References}

1. Ramanan, N. and Homsy, G.M., 1994. Linear stability of liddriven cavity flows, Phys. Fluids,6, 2690-2701.

2. Iwatsu R., Hyun J. M. and Kuwahara K., 1992. Convection in a differentially heated square cavity with a torsionallyoscillating lid, Int. J. Heat Mass Transfer, 35, 1069-1076.

3. Iwatsu R., Hyun J.M. and Kuwahara K., 1992, Numerical simulation of flows driven by a torsionally-oscillating lid in a square cavity, J. Fluids Eng,114, 143-149.

4. Waheed M.A., 2009. Mixed convective heat transfer in rectangular enclosures driven by a continuously moving horizontal plate, Int. J. Heat Mass Transfer, 52, 5055-5063.

5. Chen, C.J., Naseri-Neshat, H. and Ho, K.S., 1981. Finiteanalytical numerical solution of heat transfer in two dimensional cavity flow, Numerical Heat Transfer, 4,179-197.

6. Abu-Nada, E. and Chamkha, A.J., 2010. Mixed convection flow in a lid-driven inclined square enclosure filled with a nanofluid. European J. Mechanics B Fluids, 29, 472- 482.

7. Aydin, O., 1999. Aiding and opposing mechanisms of mixed convection in a shear and buoyancy driven cavity, Int. Comm. Heat Mass Transfer, 26, 1019-1028.

8. Ghia,U., Ghia, K.N. and Shin, C.T., 1982. High-Re Solutions for Incompressible Flow Using the Navier-Stokes Equations and a Multigrid Method. J. Comput. Physics, 48, 387- 411. 
9. Moallemi, M.K. and Jang, K.S., 1992. Prandtl number effects on laminar mixed convection heat transfer in a lid-driven cavity. Int. J. Heat Mass Transfer, 35(8), 1881- 1892.

10. Hakan, F. and Oztop, I.D. 2004. Mixed convection in twosided lid driven differentially heated square cavity. Int. J.Heat Mass Transfer, 47, 1761-1769.

11. Chamkha, A.J. 2002. Hydromagnetic combined convection flow in a vertical lid-driven cavity with internal heat generation or absorption. Numerical Heat Transfer, Part A, 41, 529-546.

12. Hussain, S. 2017. Effects of inclination angle on mixed convective nanofluid flow in a double lid-driven cavity with discrete heat sources. Int. J. Heat Mass Transfer, 106, 847860 .

13. Sivasankaran, V.S. 2013. Numerical study on mixed convection in an inclined lid-driven cavity with discrete heating. Int. Comm. Heat Mass Transfer, 46, 112- 125.

14. Sharif, M.A.R. 2007. Laminar mixed convection in shallow inclined driven cavities with hot moving lid on top and cooled from bottom, Appl. Therm. Eng, 27, 1036-1042.
15. Cheng, T.S. 2011. Characteristics of mixed convection heat transfer in a lid-driven square cavity with various Richardson and Prandtl numbers. Int. J. Thermal Sciences, 50, 197- 205.

16. Khorasanizade, S., and Sousa, J. M. M. 2014. A detailed study of lid-driven cavity flow at moderate Reynolds numbers using Incompressible SPH. Int. J. Num. Methods Fluids, 76, 653- 668.

17. Omari, R., 2016. Numerical Investigation of a Mixed Convection Flow in a Lid-Driven Cavity. American J. Computational Mathematics, 6, 251- 258.

18. Adair, D, Jaeger, M. 2015. Developing an Understanding of the Steps Involved in Solving Navier-Stokes Equations. The Mathematica Journal, 17, 1-19.

19. Malik, A.N., 2016. A comperative study of mixed convection and its effects on partially active thermal zones in a two sided lid-driven cavity filled with nanofluid. Eng. Science Technology, an Int. J., 19, 1283-1298. 
